\title{
Tratamento de hemangioma ulcerado: relato de caso
}

\author{
Treating an ulcerated hemangioma: a case report
}

\author{
Guilherme Benjamin Brandão Pitta ${ }^{1}$, Rosamaria Rodrigues Gomes ${ }^{2}$
}

\begin{abstract}
Resumo
O hemangioma é o tumor vascular mais presente em crianças até 1 ano de idade. Geralmente, sua involução é espontânea, sendo por isso recomendada uma conduta expectante. Ulceração e infecção secundária, seguidas de hemorragia, são as complicações mais frequentes dos hemangiomas. Estima-se que apenas 10 a 20\% dos hemangiomas precisem ser tratados. A cirurgia é indicada nos casos de emergência, naqueles em que não há resposta aos tratamentos sistêmicos ou por razões estéticas. $\mathrm{O}$ objetivo deste estudo é relatar um caso de hemangioma combinado ulcerado do pavilhão auricular em paciente de 8 meses de idade submetida a exérese total.
\end{abstract}

Palavras-chave: Hemangioma, neoplasias de tecido vascular, cirurgia, complicações.

\section{Introdução}

Os hemangiomas são anomalias vasculares do grupo das angiodisplasias ${ }^{1}$, sendo o tumor vascular mais comum na infância, com ocorrência de 10 a 12\% em crianças com até 1 ano de idade ${ }^{2}$.

Virchow, em 1863, foi quem classificou pela primeira vez as anomalias vasculares, com base em seu quadro microscópico, em angioma simples, cavernoso e racemoso ${ }^{3}$. Em 1982, surgiu uma nova classificação das lesões vasculares, baseada nas manifestações clínicas, quadro histopatológico e história natural, distinguindo-as em hemangiomas e malformações vasculares. Em 1996, a Sociedade Internacional para o Estudo de Anomalias Vasculares revisou essa classificação e a adotou como oficial, dividindo as lesões vasculares em dois grupos: tumores e malformações vasculares ${ }^{4}$.

Os tumores vasculares são neoplasias da vasculatura, com proliferação de células endoteliais. Incluem hemangioma da infância, hemangioma congênito rapidamente involutivo, hemangioma congênito não involutivo, angioma em tufos, hemangioendotelioma kaposiforme e granuloma

\begin{abstract}
Hemangioma is the most common vascular tumor in children younger than 12 months. Because its regression is usually spontaneous, an expectant management is recommended in most cases. Ulceration and secondary infection followed by bleeding are the most frequent complications of hemangiomas. It is estimated that only 10 to $20 \%$ of hemangiomas require treatment. Surgery is usually indicated in emergency cases, when there is no response to systemic treatments, or for cosmetic reasons. The objective of this study was to report a case of ulcerated compound hemangioma on the external ear of an 8-month-old patient who underwent a complete surgical excision.
\end{abstract}

Keywords: Hemangioma, vascular tissue neoplasms, surgery, complications.

piogênico. As malformações vasculares consistem em erros da morfogênese e são classificadas de acordo com o vaso predominante.

Clinicamente, os hemangiomas podem ser divididos em:

1) Planos: superficiais ou profundos, conhecidos como manchas tipo vinho do Porto, são as lesões mais frequentes entre as malformações vasculares, sendo evidentes desde o nascimento e podendo formar nodulações a partir da segunda década de vida.

2) Tumorais: fragiformes, tuberosos e cavernosos. Hemangiomas tumorais fragiformes e tuberosos são considerados proliferativos, observados desde o nascimento. Costumam regredir de forma espontânea a partir do segundo ano de vida, mas podem evoluir para hemangiomas alarmantes. Hemangiomas cavernosos são decorrentes de defeitos na morfogênese vascular. São malformações venosas com componente arterial e não sofrem regressão espontânea, devendo ser considerada precocemente a possibilidade de tratamento cirúrgico ${ }^{5}$.

1. Doutor. Professor adjunto, Universidade Estadual de Ciências da Saúde de Alagoas (UNCISAL), Maceió, AL.

2. Acadêmica de Medicina, UNCISAL, Maceió, AL.

Não foram declarados conflitos de interesse associados à publicação deste artigo.

Artigo submetido em 15.06.08, aceito em 30.03.09.

J Vasc Bras. 2009;8(3):263-266.

Copyright (C 2009 by Sociedade Brasileira de Angiologia e de Cirurgia Vascular 
Em qualquer fase da história natural da doença, a ulceração é sua complicação mais comum, correspondendo a $23 \%$ das complicações ${ }^{6}$, e pode demandar intervenção cirúrgica ${ }^{7}$. Outras complicações, como comprometimento visual e cardíaco, obstrução de via aérea e do conduto auditivo, representam $10,2 \%$ das complicações ${ }^{6}$.

\section{Descrição do caso}

Paciente do sexo feminino, 8 meses, residente em Recife (PE), portadora de lesão ulcerada em pavilhão auricular esquerdo, foi admitida no hospital com queixa de sangramento na orelha. Foi solicitada avaliação do cirurgião vascular. Ao exame, a paciente apresentava lesão bem delimitada, nodular, de cor vermelho vivo, com telangiectasias na superfície ulcerada, sangrante na região posterior do pavilhão auricular esquerdo no lobo inferior, com abaulamento no interior da orelha (Figura 1). Sobreposto à lesão, havia formação de abscesso local.

Não foi realizado exame complementar, haja vista o diagnóstico eminentemente clínico. Foi indicada cirurgia para exérese do tumor vascular. O procedimento cirúrgico transcorreu sob indução anestésica com sevoflurano, com entubação orotraqueal, com a paciente em decúbito dorsal. Foi realizada antissepsia e aposição dos campos cirúrgicos, seguida de incisão na face posterior do pavilhão auricular esquerdo e exérese parcial de hemangioma cavernoso. A seguir, procedeu-se a incisão dentro do pavilhão auricular e exérese total do hemangioma. Finalizando, foi realizada hemostasia, síntese e curativo da ferida operatória. A paciente evoluiu sem intercorrências no pós-operatório (Figura 2). O exame anatomopatológico revelou uma proliferação de vasos revestidos por endotélio típico em

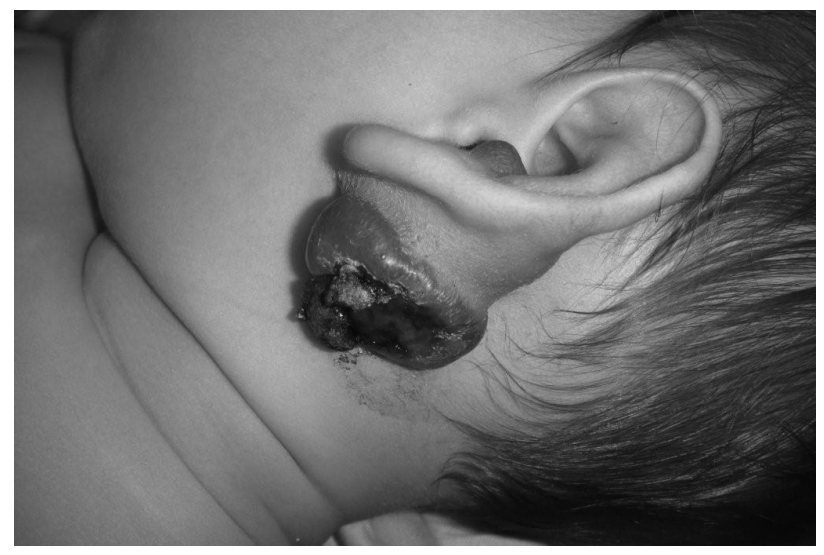

Figura 1 - Hemangioma ulcerado: pré-operatório

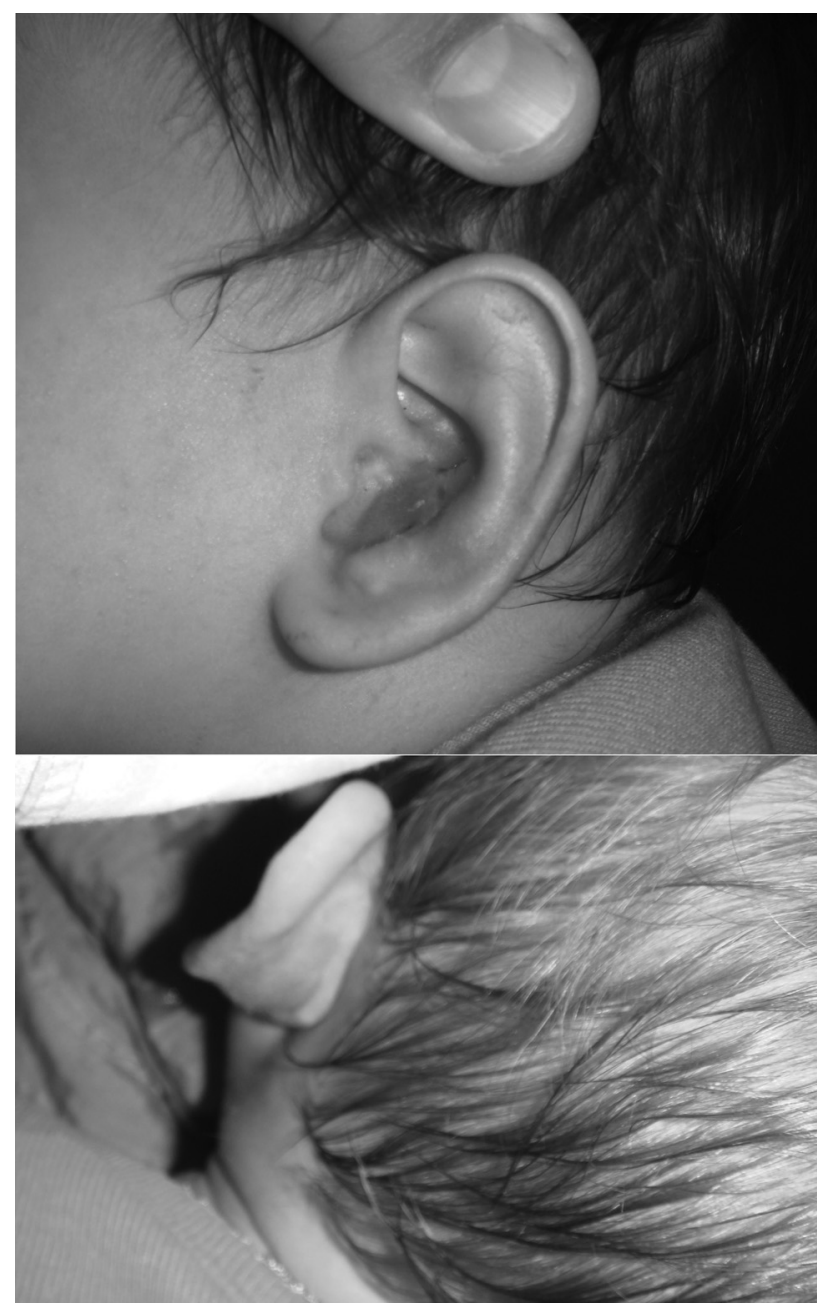

Figura 2 - Hemangioma ulcerado: pós-operatório

meio a tecido conjuntivo frouxo, com infiltrado mononuclear de permeio, confirmando o diagnóstico de hemangioma cavernoso.

\section{Discussão}

Os hemangiomas caracterizam-se por um crescimento rápido pós-nascimento seguido de involução espontânea lenta; a relação de frequência entre o sexo feminino e masculino é de $5: 1^{2}$. Os primeiros sinais da lesão são detectados no primeiro mês de vida em $90 \%$ dos casos e acometem o segmento cefálico em $73 \%{ }^{1}$.

Os primeiros meses de vida são um período crítico no crescimento do hemangioma, e crianças com esse tumor vascular requerem estreita observação, devendo ser referidas aos cuidados médicos tão logo surjam as complicações $^{8}$. Na quase totalidade dos casos, o diagnóstico do tumor pode ser realizado com base exclusivamente nos 
achados físicos e história clínica, e a conduta geralmente é expectante ${ }^{9}$.

O crescimento se deve à proliferação de células endoteliais mediada por fatores estimuladores de angiogênese ${ }^{1}$. $\mathrm{Na}$ fase de crescimento, observam-se agregados de células endoteliais proliferativas constituindo cordões sólidos e massas, por vezes com formação de lúmen; a trombose e os depósitos de hemossiderina são raros, limitados às regiões de ulceração e inflamação; as células endoteliais achatam-se na fase involutiva, com tecido adiposo e fibroso substituindo a área tumoral ${ }^{2}$.

Cerca de $24 \%$ dos pacientes com hemangiomas apresentarão complicações ${ }^{6}$. A ulceração é a complicação mais frequente ( 5 a $13 \%$ dos casos) na fase de proliferação rápi$\mathrm{da}^{7}$ e ocorre geralmente em torno dos 4 meses de vida; sangramentos acometem $41 \%$ das lesões ulceradas, e a frequência de infecções dessas lesões é de $16 \%{ }^{10}$. A infecção secundária é comum e geralmente restrita à pele, podendo acometer estruturas profundas. Sangramentos de pouca intensidade podem ocorrer e respondem bem à compressão direta, sendo raros os casos mais graves, que demandam intervenção cirúrgica ${ }^{11}$.

A frequência de complicações e a necessidade de tratamento variam de acordo com a localização do hemangioma, sendo os tumores de face e pescoço responsáveis por $43 \%$ das complicações ${ }^{6}$. A insuficiência cardíaca congestiva é uma complicação rara e pode estar associada a hemangiomas de grandes dimensões ou a hemangiomas múltiplos, sendo o tratamento direcionado para a redução do volume sanguíneo através da regressão do hemangioma. O hipotireoidismo também pode estar associado a hemangiomas gigantes, que, devido à alta vascularização e produção da enzima 3-iodotironina deiodinase, pode inativar excessivamente o hormônio tireoidiano. Alteração da visão pode ocorrer quando um hemangioma da região periorbital provoca obstrução do eixo visual, compressão do globo ocular ou quando o hemangioma se expande para o espaço retrobulbar. Pode haver comprometimento da respiração, resultante da obstrução das vias aéreas por hemangioma localizado nas fossas nasais, orofaringe ou na região traqueolaríngea. O comprometimento da audição pode ocorrer quando houver obstrução do conduto auditivo externo. Finalmente, a desfiguração pode ser uma consequência de hemangiomas grandes de face e pescoço, cujas lesões resi- duais podem deixar deformidades de difícil correção, já que nesses locais a regressão é lenta e incompleta ${ }^{2}$.

Estima-se que apenas 10 a 20\% dos hemangiomas precisem ser tratados ${ }^{9}$. Nesses se incluem todas as complicações mencionadas anteriormente. $\mathrm{O}$ tratamento deve levar em consideração a idade do paciente, o tamanho, número e localização das lesões, seu estágio evolutivo e a presença de outros sintomas associados ${ }^{12}$.

Para a maior parte dos hemangiomas, o tratamento é conservador e inclui corticoterapia (sistêmica, tópica e injeção local), interferon-alfa, laser, embolização, crioterapia e radiação ${ }^{13}$. O uso de corticoides e interferon pode causar falência adrenal; o laser pode resultar em cicatriz, atrofia ou depressão da pele, hiperpigmentação transitória ou permanente, bem como aumento dos custos para o paciente; a crioterapia também pode resultar em cicatriz e hiperpigmentação $^{13}$. A escleroterapia também é utilizada, e seus efeitos indesejáveis podem ser tromboflebite, tromboembolismo pulmonar, necrose de pele e anafilaxia ${ }^{14}$.

A cirurgia é um dos pilares do tratamento dos hemangiomas e tem indicações concretas, principalmente na fase de proliferação, sendo indicado o procedimento cirúrgico principalmente devido à deformidade estética e sua consequente influência negativa na formação da personalidade e autoestima da criança ${ }^{15}$. A cirurgia é indicada nos casos de emergência, naqueles em que não há resposta aos tratamentos sistêmicos ou por razões estéticas. Pode ser empregada sob forma de embolização, ligação arterial seletiva ou exérese simples, com ou sem reconstrução plástica ${ }^{9}$.

\section{Referências}

1. Campos HGA, Curado JH. Angiodisplasias. In: Pitta GBB, Castro AA, Burihan E, editores. Angiologia e cirurgia vascular: guia ilustrado. Maceió: UNCISAL/ECMAL \& LAVA; 2003. http://www.lava.med.br/livro.

2. Gontijo B, Silva CMR, Pereira LB. Hemangioma da infância. An Bras Dermatol. 2003;78:651-73.

3. Mulliken JB, Fishman SJ, Burrows PE. Vascular anomalies. Curr Probl Surg. 2000;37:518-84.

4. Enjolras O, Mulliken JB. Vascular tumors and vascular malformations (new issues). Adv Dermatol. 1997;13:375-422.

5. Campos HGA, Curado JH. Angiodisplasias. In: Maffei FHA, Lastória S, Yoshida WB, Rollo HA, Giannini M, Moura R. Doenças vasculares periféricas. $4^{\mathrm{a}} \mathrm{ed}$. Rio de Janeiro: Guanabara Koogan; 2008. Vol. 2, p. 1949-77.

6. Haggstrom AN, Drolet BA, Baselga E, et al. Prospective study of infantile hemangiomas: clinical characteristics predicting complications and treatment. Pediatrics. 2006;118:882-7. 
7. Garzon MC, Frieden IJ. Hemangiomas: when to worry. Pediatr Ann. 2000;29:58-67.

8. Chang LC, Haggstrom AN, Drolet BA, et al. Growth characteristics of infantile hemangiomas: implications for management. Pediatrics. 2008;122:360-7.

9. Bruckner AL, Frieden IJ. Hemangiomas of infancy. J Am Acad Dermatol. 2003;48:477-93.

10. Chamlin SL, Haggstrom AN, Drolet BA, et al. Multicenter prospective study of ulcerated hemangiomas. J Pediatr. 2007;151:684-9.

11. Esterly NB. Haemangiomas. In: Harper J, Oranje A, Prose N, editors. Textbook of pediatric dermatology. Oxford: Blackwell Science; 2000. p. 997-1016.

12. Frieden IJ. Management of hemangiomas. Special symposium. Pediatr Dermatol. 1997;14:57-83.
13. Selim H, Selim A, Khachemoune A, Metwally SA. Use of sclerosing agent in the management of oral and perioral hemangiomas: review and case reports. Med Sci Monit. 2007;13:114-9.

14. Belzunce A, Casellas M. Complications in the evolution of haemangiomas and vascular malformations. An Sist Sanit Navar. 2004;27 Suppl 1:57-69.

15. Waner M, Murillo J, Aubá C, Hontanilla B. Surgical treatment of haemangiomas. An Sist Sanit Navar. 2004;27 Suppl 1:93-101.

Correspondência:

Rosamaria Rodrigues Gomes

Rua Prof. Vital Barbosa, 996/304, Ponta Verde

CEP 57035-400 - Maceió, AL

Tel.: (82) 8812.1976

E-mail: email@rosamariagomes.com 